\title{
Simultaneous paired associate and verbal discrimination learning as a simulation of the double-function list
}

\author{
ELAINE A. BOULAY and BENTON J. UNDERWOOD \\ Northwesterm University, Evanston, Ilinois 60201
}

\begin{abstract}
In the critical condition, the subject was required to learn associations between words in the pairs and to learn to identify simultaneously the word in each pair that had been underlined on study trials. Over trials, both types of responding were required for each pair. Control conditions were used to assess the influence of these procedures. Paired associate learning was only modestly impaired, but the verbal discrimination learning (identifying the underlined word) appeared comparable to that for a double-function verbal discrimination list. The correlational evidence indicated that insofar as the present procedures simulated a double-function list, the association between the words in a pair is not involved in a causal way for the small amount of learning that occurs across trials in a double-function list.
\end{abstract}

In learning the usual verbal discrimination task, an association develops between the two words in each pair (e.g., Zechmeister \& Underwood, 1969). The development may be viewed as resulting from intentional or incidental learning. In either case, the correlation between associative formation and verbal discrimination performance might be used for inferring a cause-effect relationship. For the usual verbal discrimination list. the cumulative evidence would seem to deny a fundamental role to the association (Zimmerman, Shaughnessy, \& Underwood, 1972). There is, however, a special form of the verbal discrimination task, called the double-function list, in which the association is still sometimes inferred to play a causal role. In the double-function list, each item is used as a correct word in one pair and as an incorrect word in another pair. Such a list is very difficult to learn, but some learning by some subjects does occur (Kausler \& Boka, 1968; Underwood \& Reichardt, 1975). Because it can be shown that those subjects who do learn the task have higher associative learning than those who learn very little. the association has again been suggested as a causal mechanism. Thus, Mueller, Kausler, Yadrick, and Pavur (1975) conclude that "the association between the right and wrong terms seems implicated" (p. 59).

The procedures for the central condition of the present experiment will now be described, followed by the statement of purpose viewed in light of the above background. The subject was presented a list of 16 pairs for alternate study and test trials. On the study trials. one word in each pair was underlined. The subject was informed that the underlined word was the correct word in the pair and that when the pair was presented on the test trial without the underlining he should be able to call out the correct word. This was. therefore, a verbal discrimination task. However, the subject was also instructed that for some of the pairs only one of the words would be shown on the test trial and that he was to learn to be able to give the other word in the pair. Because the task required the subject to respond with either word, given the other, it was a bidirectional paired associate task. Across trials, a given pair was tested equally often for discriminative learning and for associative learning.

The above procedure was designed to simulate a double-function verbal discrimination list in which the influence of the intrapair associations could be assessed directly. The construction of the task was based on the assumption that the double-function list was difficult to learn because the use of an item in both correct and incorrect roles does not allow a frequency differential to serve as the basis of the discrimination. If the subject is required to learn a bidirectional paired associate task, the situational frequency of each word in the pair should be roughly equivalent. Therefore. it should be impossible to make frequency discriminations between the correct and incorrect words in the pairs and the performance on the verbal discrimination task embedded within the paired associate task should show little improvement over trials. The development of intrapair associations should occur regardless of the performance shown on the verbal discrimination tests.

Given that the procedures are successful in simulating a double-function list, certain other evidence may be examined to determine if the intrapair associations are critically involved in the small amount of learning which occurs for the double-function list. The most important evidence would lie in the item correlations. If the intrapair association is critically involved, there should be a substantial positive correlation across trials for paired 


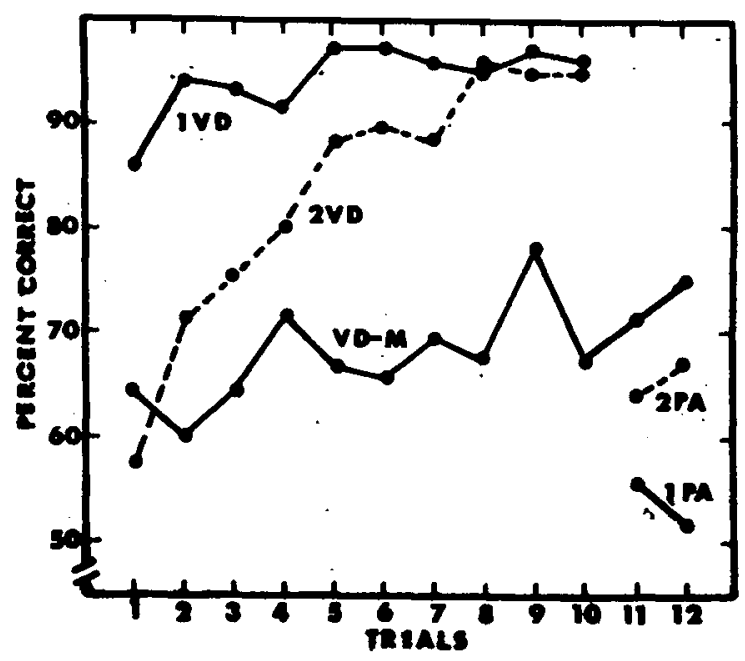

Figure 1. Acquisition curves for verbal discrimination leaming. Groups 2PA and 1PA were tested on verbal discrimination on Trials 11 and 12 only following 10 paired associate trials.

associate learning and verbal discrimination learning when the measure is the number of correct responses for each pair for each form of learning summed over subjects.

\section{METHOD}

\section{Lists and Conditions}

All subjects in all conditions were presented the same list of 16 pairs of two-syllable nouns. The words varied in frequency from 30 10.50 in the Thorndike-Lorge (1944) count. One word in each pair was randonly designated correct for the verbal discrimination learning and was underlined on all study trials. The order of the pairs varied randomly across trials. The two words in a pair were shown one above the other on study trials. with the particular word occupying the upper position changing randomly over trials. When the test trial involved paired associate responding, the particular word used as the stimulus term varied from trial to trial in order to make the learning bidirectional. The presentation of the list on the study trials was exactly the same for all groups. Each pair was presented for $2 \mathrm{sec}$ for study, and tests were also conducted at a 2 -sec rate. The groups differed in the nature of the instructions given prior to learning and in the nature of the test trials. There were five groups. and. in the symbols used to identify them, PA and $V D$ are used to identify the two tasks, with the numerals 1 and 2 indicating that the subject was instructed for one form of learning or two forms of learning. The critical group. Group M (mixed), had simultaneous PA and VD learning.

Group $M$. The test trials for this group required responding to indicate paired associate learning for some pairs on a trial. and responding to indicate verbal discrimination learning (choosing the word und ciined on the study trials) for the other pairs. There were 12 study-test trials, and across these 12 trials each item was tested six times for paired associate learning and six times for verbal discrimination learning. The particular type of test given an item on a trial was determined randomly subject only to the restriction that each item be tested six times each for the two types of learning. When an item was tested for associative learning. one of the words from the pair was shown and the subject was asked to try to say aloud the other member of the pair. When verbal discrimination testing was used, the pair was presented and the subject was asked to speak the word that had been underlined on the study trials. Forced responding was used for all tests of discrimination learning. The subjects were fully instructed concerning the nature of the task and the nature of the tests, and these instructions were implemented by using sample items on index cards during the instructions.

Group 2PA. This group received exactly the same instructions as did Group $M$. However, for the first 10 trials, all pairs were tested for paired associate learning. After the 10th trial, the subjects were in formed that no further study trials would be given. Immediately thereafter. they were given two successive verbal discrimination test trials on all 16 pairs.

Group 2VD. This group was also given the same instructions as was Group $M$, but the first 10 test trials were always verbal discrimination tests for all pairs. Following the 10th test trial, two paired associate test trials were given for all 16 pairs.

Group 1PA. This group constituted the basic control for paired associate learning. The subjects were instructed only for paired associate learning and were so tested for the first 10 trials. The instructions told the subjects to ignore the fact that one of the words in each pair was underlined. After the 10th test trial, the subjects were told: "I know that I told you to ignore the underlining, but now I want to see how well you remembered which words were underlined." Brief instructions for verbal discrimination responding were then given, followed by two test trials.

Group IVD. This group constituted the basic control for verbal discrimination learning. Initial instructions were given only for verbal discrimination learning, after which 10 study-test trials occurred. After the 10th trial, the subject was told: "I want to see how well you remember which words always appeared together in each pair." Brief instructions were then given for paired associate responding, after which two test trials were administered.

\section{Subjects}

Each of the five groups was represented by 15 college student subjects assigned to groups by a block-randomized schedule of the five conditions.

\section{RESULTS}

\section{Verbal Discrimination Leaming}

Because the number of items tested for verbal discrimination learning and for paired associate learning differed from trial to trial for Group $M$, it was necessary to use percentage scores. Therefore, scores for all groups were expressed as percentages and the statistical analyses used percentages as entries. A .05 level of significance was used throughout.

Learning curves for the verbal discrimination task are shown in Figure 1. The subjects in Group 1VD, instructed only for verbal discrimination learning, had over $85 \%$ correct responses on the first test trial. and performance over the remaining trials remained close to asymptote. Nine of the 15 subjects had all items correct on a trial during the first three trials. The performance of the subjects in Group 2VD, in which instructions to expect both tests were given, was inferior $(83.7 \%$ correct over 10 trials) to that of the subjects in Group IVD $(94.3 \%), F(1,28)=18.78$, $M S_{e}=45.45$. The critical condition is the verbal discrimination performance for Group $M$. As may be seen in Figure 1, the performance of this group, while above chance on all trials, improved very little across trials. In fact, the apparent small improvement was not reliable statistically, $\mathrm{F}(11,154)=1.31, \mathrm{MS}_{\mathrm{e}}=$ 
300.23. This condition was intended to simulate a double-function list, and it appears to have done so. If there is a difference, it lies in the fact that performance on the usual double-function list is closer to chance on the early trials (e.g., Underwood \& Reichardt, 1975). Of the 15 subjects, 8 achieved a perfect performance on at least one trial. Perfect performance on a given trial was frequently followed by deteriorated performance on the following trial.

It will be remembered that Group 1PA was given two verbal discrimination test trials following 10 paired associate trials. The fact that performance is only slightly above chance indicates that essentially no incidental learning of the underlined word occurred. The subjects in Group 2PA had been told to expect verbal discrimination tests, although they were not so tested until the last two trials. Figure 1 shows that the performance of this group is below that of Group $M$, although the difference in the means $(65.3 \%$ vs. $73.9 \%$ ) was not reliable, $\mathrm{F}(1,28)=1.71, \mathrm{MS}_{\mathrm{e}}=$ 324.45. Some difference should be expected on the basis of the fact that the subjects in Group $M$ had study trials before their tests on Trials 11 and 12 , whereas the subjects in Group 2PA were given only test trials on 11 and 12 .

\section{Paired Associate Learning}

The trial-by-trial scores for paired associate learning are shown in Figure 2. Across the 10 trials, Groups 2PA and 1PA did not differ in performance. Thus, expectation of being tested on verbal discrimination learning did not influence paired associate performance, whereas, as noted above and as seen in Figure 1, expectation of being tested on paired associate learning severely retarded verbal discrimination learning. The reason for the interaction across trials for Groups 1PA and 2PA is not known.

The paired associate performance of the subjects in Group $M$ appears depressed when judged against the control (Group 1PA). Statistically speaking, the difference between the two groups in percentage correct responses across the 10 trials $(66.4 \%$ vs. $50.1 \%$ ) falls short of significance, $F(1,28)=3.69$, $\mathrm{MS}_{\mathrm{e}}=542.30$. Nevertheless, the consistency with which the two curves differ across trials may argue for reliability. However this may be, it is quite evident that the depression in paired associate learning for the subjects in Group $M$ is far less than the depression in verbal discrimination performance for these same subjects. It may also be pointed out that the performance on the two paired associate test trials for subjects in Group 2VD was essentially as good as was the performance for the subjects in Group $M$. The performance of Group 2VD $(65.9 \%$ correct) was superior to that of Group IVD $(36.0 \%), F(1,28)=$ 11.77. $\mathrm{MS}_{\mathrm{e}}=570.75$.

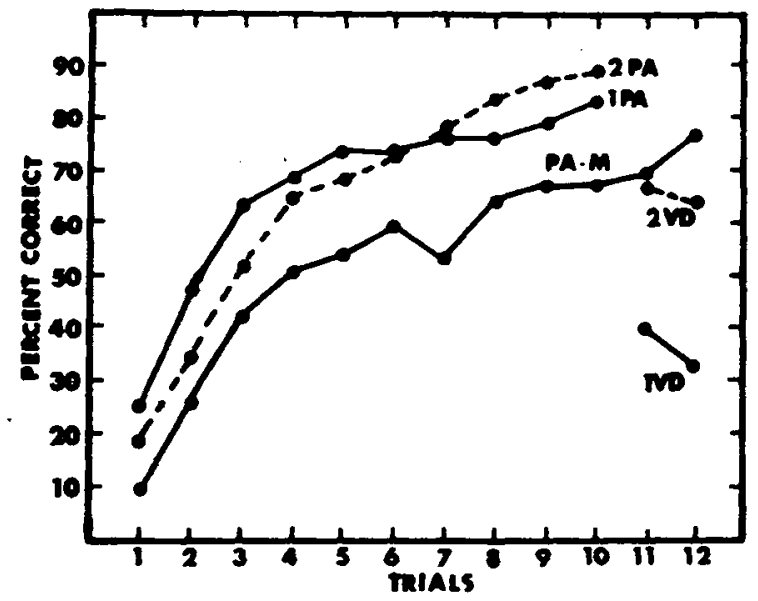

Figure 2. Acquisition curves for paired associate learning. Groups 2VD and IVD were teated on paired associate learning on Trials 11 and 12 only following 10 trials of verbal discrimination leaming.

Figure 1 showed that the subjects in Group IPA had acquired little, if any, verbal discrimination knowledge. Figure 2 shows that a considerable amount of associative learning occurred incidentally for the subjects in Group IVD. This confirms many earlier studies which have shown that the development of intrapair associations during verbal discrimination learning seems inevitable.

\section{Correlational Evidence}

Studies which have shown a correlation across subjects between learning a double-function list and incidental associative learning (e.g., Mueller et al., 1975) have led to the suggestion that associative learning may be causally involved in the meager learning that occurs in the double-function list. For the subjects in Group $M$, the product moment correlation between the total number of correct responses across 12 trials for verbal discrimination tests and for paired associate tests was .71. The present data therefore replicate earlier results on this matter. A sampling of trials showed that this positive relationship developed quite early in learning. For Trials $1,3,5,8$, and 12 , the values were $-.12, .51, .55$, .68 . and .66 . All of this evidence indicates, therefore, that a subject whose performance was high on one of the tasks was likely to have high performance on the other.

The problem with tracing the implications of the above correlation is, of course, that a subject may be proficient on both tasks for quite different reasons. A general learning ability may underlie the performance on both tasks. A more compelling case can be made by examining performance as a function of items. If the association is involved in a causal way in learning the double-function list, the pairs for which associations are quickly developed should also 
constitute the easy pairs for verbal discrimination learning. The total number of correct responses for the verbal discrimination tests (summed across subjects) and for the paired associate tests were determined for each pair for Group $M$. The product moment correlation for these two arrays was -.13. There appears to be no relationship between paired associate learning and verbal discrimination learning for these 16 pairs. This lack of a relationship is not due to the low reliability of item difficulty of the pairs in paired associate learning. The number of correct responses for each of the pairs summed across trials and subjects was determined independently for Groups 1PA. 2PA, and $M$. The three possible correlations among these groups were $.63, .64$, and .68 . Item difficulty was not disturbed in Group $M$ as a consequence of the attempted simultaneous learning of the verbal discriminations.

As a final test, the item correlations were examined for individual subjects in Group M. Each pair had six tests on associative learning and six tests on discrimination learning. The 15 correlations varied from .40 to -.26 . Six were positive, nine were negative, and none was statistically different from zero.

\section{DISCUSSION}

The goal of producing a double-function verbal discrimination task by requiring subjects to learn a bidirectional paired associate task appears to have been successful. At best, paired associate learning was impaired only a small amount, while verbal discrimination learning was seriously impaired. This outcome would be anticipated if a frequency differential was normally the basis on which the subject discriminated between right and wrong words in the pairs. The bidirectional paired associate learning equated roughly the situational frequency of the right and wrong words and, as in the usual double-function list, there appears to be no other consistent basis for a discrimination which the subject can employ.

The correlational evidence is viewed as indicating that it is highly unlikely that the intrapair associations are involved in a causal way in the small amount of learning observed in the usual double-function list. The question as to how a subject makes any progress in learning a double-function list remains to be discovered.

\section{REFERENCES}

KAusler, D. H., \& BoKA, J. A. Effects of double functioning on verbal-discrimination learning. Journal of Experimental Psychology, 1968, 76, 558-567.

Mueller, J. H., Kausler, D. H., Yadrick, R. M., \& Pavur, E. J. Encoding strategies in double-function verbal discrimination learning. Journal of Experimental Psychology: Human Learning and Memory. 1975. 104, 55-59.

THORNDIKE, E. L.. \& LORGE, I. The teacher's word book of 30.000 words. New York: Teachers College, Columbia University, 1944.

UNDERWoOd, B. J., \& ReIchardt, C. S. Contingent associations and the double-function, verbal-discrimination task. Memory \& Cognition, 1975, 3. 311-314.

Zechmeister, E. B., \& Underwood, B. J. Acquisition of items and associations in verbal discrimination learning as a function of level of practice. Journal of Experimental Psychology, 1969, 81. 355-359.

Zimmerman, J., Shaughnessy, J. J.. \& Underwood, B. J. The role of associations in verbal-discrimination learning. American Journal of Psychology, 1972, 85, 499-518.

(Received for publication July 31. 1975; revision received October 6, 1975.) 